T. H. Steele,Department of Mathematics, Weber State University, Ogden, UT, 84408-1702, USA. e-mail: thsteele@weber.edu

\title{
THE PERSISTENCE OF $\omega$-LIMIT SETS UNDER PERTURBATION OF THE GENERATING FUNCTION
}

\begin{abstract}
We consider the set valued function $\Omega$ taking $f$ in $C(I, I)$ to its collection of $\omega$-limit sets $\Omega(f)=\{\omega(x, f): x \in I\}$, and consider how $\Omega(f)$ is affected by pertubations of $f$. Our main result characterizes those functions $f$ in $C(I, I)$ at which $\Omega$ is upper semicontinuous, so that whenever $g$ is sufficiently close to $f$, every $\omega$-limit set of $g$ is close to some $\omega$-limit set of $f$ in the Hausdorff metric space. We also develop necessary and sufficient conditions for a function $f$ in $C(I, I)$ to be a point of lower semicontinuity of the map $\Omega$.
\end{abstract}

\section{Introduction}

At the Twentieth Summer Symposium in Real Analysis, A. M. Bruckner posed several questions regarding the iterative stability of continuous functions as they undergo small perturbations, as well as why these questions are of general interest [B]. In particular, how are the set of $\omega$-limit points and the collection of $\omega$-limit sets of a function affected by slight changes in that function? As Bruckner discusses in $[\mathrm{B}]$, we may also want to ask these questions when restricting our attention to particular subsets of $C(I, I)$, such as those functions that are in some way nonchaotic, or those functions that satisfy a particular smoothness condition. As one sees from various examples found in $[\mathrm{B}]$ and $[\mathrm{TH}]$, in general, both the set of $\omega$-limit points and the collection of $\omega$-limit sets of a typical function are affected dramatically by arbitrarily small perturbations. In [TH2] we make some progress towards understanding the continuity structure of the maps $f \mapsto \cup_{x \in I} \omega(x, f)$ and $f \mapsto\{\omega(x, f): x \in I\}$. We take $(K, H)$ to be the class of nonempty closed sets $K$ in $I$ endowed with the Hausdorff metric $H$, and let $\left(K^{*}, H^{*}\right)$ consist of the nonempty closed subsets of $K$. We are then

Key Words: $\omega$-limit set, topological entropy, semicontinuity

Mathematical Reviews subject classification: 26A18, 54C60, 26E25

Received by the editors February 3, 2001 
able to characterize those functions at which $\Lambda:(C(I, I),\|\circ\|) \rightarrow(K, H)$ given by $f \mapsto \Lambda(f)=\cup_{x \in I} \omega(x, f)$ is continuous, as well as characterize the points of continuity of the map $\Omega:(C(I, I),\|\circ\|) \rightarrow\left(K^{*}, H^{*}\right)$ given by $f \mapsto \Omega(f)=\{\omega(x, f): x \in I\}$ when we restrict the domain of $\Omega$ to those continuous functions possessing zero topological entropy. These results are presented in the following two theorems.

Theorem 1.1. The map $\Lambda:(C(I, I),\|\circ\|) \rightarrow(K, H)$ is continuous at $f$ if and only if the stable periodic points of $f$ are dense in the set of chain recurrent points of $f$.

Theorem 1.2. Let $E=\{f \in C(I, I): \mathbf{h}(f)=0\}$. Then $\Omega:(E,\|\circ\|) \rightarrow$ $\left(K^{*}, H^{*}\right)$ is continuous at $f$ if and only if either of the following equivalent conditions hold:

1. The stable periodic points of $f$ are dense in the set of chain recurrent points of $f$.

2. Every periodic point of $f$ is stable, and every simple system of $f$ has nonempty interior.

It is interesting to note that if a continuous function possesses zero topological entropy, then $\Omega:(E,\|\circ\|) \rightarrow\left(K^{*}, H^{*}\right)$ is continuous there if and only if $\Lambda:(C(I, I),\|\circ\|) \rightarrow(K, H)$ is continuous there. In this paper we build upon the results of [TH2] as we investigate the continuity structure of $\Omega$ without any domain restrictions. In particular, we develop necessary and sufficient conditions for $\Omega$ to be lower semicontinuous at $f$, and characterize those functions at which $\Omega$ is upper semicontinuous.

We proceed through several sections. In section 2 we present the notation and definitions we will use throughout the balance of the paper. Section 3 is dedicated to the development of necessary and sufficient conditions on a function $f \in C(I, I)$ which insure that it is a point of lower semicontinuity of $\Omega:(C(I, I),\|\circ\|) \rightarrow\left(K^{*}, H^{*}\right)$, and in section 4 we characterize those functions at which $\Omega$ is upper semicontinuous. We conclude our work in section 5 with a few observations and a brief discussion of open problems.

\section{Preliminaries}

We shall be concerned with the class $C(I, I)$ of continuous functions mapping the unit interval $I=[0,1]$ into itself, and the iterative properties this class of functions possesses. For $f$ in $C(I, I)$ and any integer $n \geq 1, f^{n}$ denotes the $n^{\text {th }}$ iterate of $f$. Let $P(f)$ represent those points $x \in I$ that are periodic 
under $f$, and if $x$ is a periodic point of period $n$ for which $f^{n}(x)-x$ is not unisigned in any deleted neighborhood of $x$, then $x$ is called a stable periodic point; we let $S(f)$ represent the stable periodic points of $f$. For each $x$ in $I$, we call the set of all subsequential limits of the sequence $\left\{f^{n}(x)\right\}_{n=0}^{\infty}$ the $\omega$-limit set of $f$ generated by $x$, and write $\omega(x, f)$. Let $\Lambda(f)=\cup_{x \in I} \omega(x, f)$ represent the $\omega$-limit points of $f$, while $\Omega(f)=\{\omega(x, f): x \in I\}$ denotes the set composed of the $\omega$-limit sets of $f$. Now, let $\varepsilon>0$ be given, and take $x$ and $y$ to be any points in $[0,1]$. An $\varepsilon$-chain from $x$ to $y$ with respect to a function $f$ is a finite set of points $\left\{x_{0}, x_{1}, \ldots, x_{n}\right\}$ in $[0,1]$ with $x=x_{0}, y=x_{n}$ and $\left|f\left(x_{k-1}\right)-x_{k}\right|<\varepsilon$ for $k=0,1, \ldots, n-1$. We call $x$ a chain recurrent point of $f$ if there is an $\varepsilon$-chain from $x$ to itself for any $\varepsilon>0$, and write $x \in C R(f)$. We note that for every $f$ in $C(I, I), \Lambda(f) \subseteq C R(f)$.

In addition to the usual, Euclidean metric $d$ on $I=[0,1]$, we will be working in three metric spaces. Within $C(I, I)$ we will use the supremum metric given by $\|f-g\|=\sup \{|f(x)-g(x)|: x \in I\}$. Our second metric space $(K, H)$ is composed of the class of nonempty closed sets $K$ in $I$ endowed with the Hausdorff metric $H$ given by $H(E, F)=\inf \left\{\delta>0: E \subset B_{\delta}(F), F \subset B_{\delta}(E)\right\}$, where $B_{\delta}(F)=\{x \in I: d(x, y)<\delta, y \in F\}$. This space is compact [BBT]. Our final metric space $\left(K^{*}, H^{*}\right)$ consists of the nonempty closed subsets of $K$. Thus, $K \in K^{*}$ if $K$ is a nonempty family of nonempty closed sets in $I$ such that $K$ is closed in $K$ with respect to $H$. We endow $K^{*}$ with the metric $H^{*}$ so that $K_{1}$ and $K_{2}$ are close with respect to $H^{*}$ if each member of $K_{1}$ is close to some member of $K_{2}$ with respect to $H$, and vice versa. This metric space is also compact [B]. Our interest in, and the utility of, the spaces $(K, H)$ and $\left(K^{*}, H^{*}\right)$ stem from the following two theorems from [BCl] and [BBHS], respectively.

Theorem 2.1. For any $f$ in $C(I, I)$, the set $\Lambda(f)$ is closed in $I$.

Theorem 2.2. For any $f$ in $C(I, I)$, the set $\Omega(f)$ is closed in $K, H)$.

To a large extent, the work of $[\mathrm{B}],[\mathrm{TH}]$ and [TH2] investigates the iterative stability of $f \in C(I, I)$ under small perturbations by studying the continuity structure of the maps $\Lambda:(C(I, I),\|\circ\|) \rightarrow(K, H)$ given by $f \mapsto \Lambda(f)$, and $\Omega:(C(I, I),\|\circ\|) \rightarrow\left(K^{*}, H^{*}\right)$ given by $f \mapsto \Omega(f)$. In this paper we continue that work by focusing on the semicontinuity of $\Omega:(C(I, I),\|\circ\|) \rightarrow\left(K^{*}, H^{*}\right)$.

In part of the sequel we will restrict our attention to a closed subset $E$ of $C(I, I)$ composed of those functions $f$ having zero topological entropy, denoted by $\mathbf{h}(f)=0$. The reader is referred to Theorem A of [FSS] for an extensive list of equivalent formulations of topological entropy zero. For our purposes, it suffices to note that every periodic orbit of a continuous function with zero topological entropy has cardinality of a power of two. The following theorem, 
due to Smital [S], sheds considerable light on the structure of infinite $\omega$-limit sets for functions with zero topological entropy.

Theorem 2.3. If $\omega$ is an infinite $\omega$-limit set of $f \in C(I, I)$ possessing zero topological entropy, then there exists a sequence of closed intervals $\left\{J_{k}\right\}_{k=1}^{\infty}$ in $[0,1]$ such that

1. for each $k,\left\{f^{i}\left(J_{k}\right)\right\}_{i=1}^{2^{k}}$ are pairwise disjoint, and $J_{k}=f^{2^{k}}\left(J_{k}\right)$;

2. for each $k, J_{k+1} \cup f^{2^{k}}\left(J_{k+1}\right) \subset J_{k}$;

3. for each $k, \omega \subset \cup_{i=1}^{2^{k}} f^{i}\left(J_{k}\right)$, and

4. for each $k$ and $i, \omega \cap f^{i}\left(J_{k}\right) \neq \emptyset$.

We make the following definitions with Smital's Theorem in mind. Let $\omega$ be an infinite compact subset of $I$, and let $f$ map $\omega$ into itself. We call $f$ a simple map on $\omega$ if $\omega$ has a decomposition $S \cup T$ into compact portions that $f$ exchanges, and $f^{2}$ is simple on each of these portions. From Smital's Theorem one sees that every map $f$ with zero topological entropy is simple on each of its infinite $\omega$-limit sets. Let $\left\{J_{k}\right\}_{k=1}^{\infty}$ be a nested sequence of compact periodic intervals with respect to $\omega$ and $f$ as described in Smital's Theorem. Every set of the form $\omega \cap f^{i}\left(J_{k}\right)$ is periodic of period $2^{k}$, and we call each such set a periodic portion of rank $k$. This system of periodic portions of $\omega$, or of the corresponding periodic intervals, is called the simple system of $\omega$ with respect to $f$. We now recall a device from [D] that allows us to code the sets $f^{i}\left(J_{k}\right)$ with finite tuples of zeros and ones. Let $\mathbb{N}$ denote the natural numbers, and take $N$ to be the set of sequences composed of zeros and ones. If $\mathbf{n} \in N$ and $\mathbf{n}=\left\{n_{i}\right\}_{i=1}^{\infty}$, we let $\mathbf{n} \mid k=\left(n_{1}, n_{2}, \ldots, n_{k}\right)$. Set $\mathbf{0}=(0,0, \ldots$. and $\mathbf{1}=(1,1, \ldots$.$) . Now, define a function A: N \rightarrow N$ given by $A(\mathbf{n})=\mathbf{n}+1 \mathbf{0}$, where addition is modulus two from left to right. For each $k \in \mathbb{N}$ and $i \in \mathbb{N}$ put $F_{\mathbf{1} \mid k}=J_{k}$ and $F_{A^{i}(\mathbf{1} \mid k)}=f^{i}\left(J_{k}\right)$. Thus, for each $\mathbf{m}$ and $\mathbf{n}$ in $N$ and $k \in \mathbb{N}$ there is a $j \in \mathbb{N}$ such that $A^{j}(\mathbf{m} \mid k)=\mathbf{n} \mid k$; the above relations define $F_{\mathbf{n} \mid k}$ for all $\mathbf{n} \in N$ and $k \in \mathbb{N}$. Now, set $F_{\mathbf{n}}=\cap_{k=1}^{\infty} F_{\mathbf{n} \mid k}$, and let $K=\cup_{\mathbf{n} \in N} \cap_{k=1}^{\infty} F_{\mathbf{n} \mid k}$. Then $K$ and each $F_{\mathbf{n}}$ are compact, and the components of $K$ consist of the $F_{\mathbf{n}}$ sets.

We also need the following characterization of $\omega$-limit sets for continuous functions found in $[\mathrm{ABCP}]$.

Theorem 2.4. Let $F \subseteq I$ be a nonempty closed set. Then $F$ is an $\omega$-limit set for some $f \in C(I, I)$ if and only if $F$ is either nowhere dense, or $F$ is the union of finitely many nondegenerate closed intervals. 


\section{Lower Semicontinuity}

We begin with a definition. Suppose $f_{n} \rightarrow f$ in $C(I, I)$. Since $\left(K^{*}, H^{*}\right)$ is compact, there exists a subsequence $\left\{f_{n_{k}}\right\} \subseteq\left\{f_{n}\right\}$ with the property that $\lim _{k \rightarrow \infty} \Omega\left(f_{n_{k}}\right)$ exists in $\left(K^{*}, H^{*}\right)$; say $\lim _{k \rightarrow \infty} \Omega\left(f_{n_{k}}\right)=L$. We say that our map $\Omega:(C(I, I),\|\circ\|) \rightarrow\left(K^{*}, H^{*}\right)$ is lower semicontinuous at $f$ if $f_{n} \rightarrow f$ in $C(I, I)$ and $\Omega\left(f_{n}\right) \rightarrow L$ in $\left(K^{*}, H^{*}\right)$ implies $L \supseteq \Omega(f)$. It follows, then, that the lower semicontinuity of $\Lambda:(C(I, I),\|\circ\|) \longrightarrow(K, H)$ is a necessary condition for the lower semicontinuity of $\Omega:(C(I, I),\|\circ\|) \longrightarrow\left(K^{*}, H^{*}\right)$. In [TH2] we characterize the functions at which $\Lambda$ is lower semicontinuous as those functions $f$ for which $\overline{S(f)}=\Lambda(f)$. As an immediate corollary, then, we have the following.

Proposition 3.1. A necessary condition for the function $f$ to be a point of lower semicontinuity of the map $\Omega:(C(I, I),\|\circ\|) \longrightarrow\left(K^{*}, H^{*}\right)$ is that $\overline{S(f)}=\Lambda(f)$.

Our following example shows, however, that the condition of our proposition is not sufficient to insure the lower semicontinuity of $\Omega:(C(I, I),\|\circ\|) \longrightarrow$ $\left(K^{*}, H^{*}\right)$ at $f$.

Example 3.2. Let $h \in C(I, I)$ be the hat map given by $h(x)=2 x$ for $0 \leq x \leq$ $\frac{1}{2}$, and $h(x)=2(1-x)$ for $\frac{1}{2} \leq x \leq 1$. The $\operatorname{map} \Omega:(C(I, I), \|$ o $\|) \longrightarrow\left(K^{*}, H^{*}\right)$ is not lower semicontinuous at $h$ even though $\overline{S(h)}=\Lambda(h)$.

Proof. That $\overline{S(h)}=\Lambda(h)$ follows from our definition of $h$; the details are worked out in [TH2]. We now note that $h(0)=0$, so that $\omega(0, h)=\{0\}$. Define $f_{n} \in C(I, I)$ so that $f_{n}(0)=\frac{1}{n}, f_{n}\left(\frac{1}{2}\right)=1, f_{n}(1)=0$, and $f_{n}$ is linear on both $\left(0, \frac{1}{2}\right)$ and $\left(\frac{1}{2}, 1\right)$. Then $\left\|h-f_{n}\right\|=\frac{1}{n}$ for all $n \in \mathbb{N}$. Let $n \geq 2$. If $x \in \omega \in \Omega\left(f_{n}\right)$ and $0 \leq x \leq \frac{1}{2}$, then there exists $y$ in $\omega$ so that $y \geq \frac{1}{2}$. It follows that $f_{n} \rightarrow h$ in $C(I, I)$, but $H(\{0\}, \omega) \geq \frac{1}{2}$ for any $\omega$ in $\cup_{n=2}^{\infty} \Omega\left(f_{n}\right)$.

In a similar manner one also may show that $H(\{0\}, \omega) \geq \frac{1}{2}$ for any $\omega \in$ $\Omega(h)$ distinct from the unstable fixed point $\{0\}$; that is, $\{0\}$ is an unstable periodic orbit of $h$ that is isolated in $\Omega(h)$. In Proposition 3.3 we develop an analogue to the condition found in Proposition 3.1 that precludes this difficulty; we consider those continuous functions $f$ that have a dense set of stable periodic orbits in $\Omega(f)$. Since $\Omega(f)$ is closed in the compact space $\left(K^{*}, H^{*}\right)$, stable periodic orbits being dense in $\Omega(f)$ is equivalent to requiring that for any $\varepsilon>0$ there exists an $\varepsilon$-net of $\Omega(f)$ comprised entirely of stable periodic orbits.

Proposition 3.3. Let $f \in C(I, I)$. If the stable periodic orbits of $f$ are dense in $\Omega(f)$, then $\Omega:(C(I, I),\|\circ\|) \longrightarrow\left(K^{*}, H^{*}\right)$ is lower semicontinuous at $f$. 
Proof. It suffices to show that for every $\varepsilon>0$ there exists a corresponding $\delta>0$ so that $\Omega(f) \subseteq B_{\varepsilon}(\Omega(g))$ whenever $\|f-g\|<\delta$. To this end, let $\varepsilon>0$, with $\left\{\omega_{1}, \omega_{2}, \ldots, \omega_{n}\right\} \subseteq \Omega(f)$ an $\frac{\varepsilon}{2}$-net of $\Omega(f)$, where $\omega_{i} \subseteq S(f)$ for $1 \leq i \leq n$. From the definition of a stable periodic orbit, there exists $\delta_{i}>0$ so that $H\left(\omega_{i}, \alpha_{i}\right)<\frac{\varepsilon}{2}$ for some $\alpha_{i} \in \Omega(g)$ whenever $\|f-g\|<\delta_{i}$, for $1 \leq i \leq n$. Let $\delta>0$ so that $\delta<\min \left\{\delta_{1}, \delta_{2}, \ldots, \delta_{n}\right\}$, with $\omega \in \Omega(f)$ and $g \in C(I, I)$ for which $\|f-g\|<\delta$. Since $\left\{\omega_{1}, \omega_{2}, \ldots, \omega_{n}\right\}$ is an $\frac{\varepsilon}{2}$-net of $\Omega(f), H\left(\omega, \omega_{i}\right)<\frac{\varepsilon}{2}$ for some $1 \leq i \leq n$; since $\|f-g\|<\delta<\delta_{i}, H\left(\omega_{i}, \alpha\right)<\frac{\varepsilon}{2}$ for some $\alpha \in \Omega(g)$. It follows that $H(\omega, \alpha)<\varepsilon$, and in general, that $\Omega(f) \subseteq B_{\varepsilon}(\Omega(g))$.

With Proposition 3.3, we are able to develop part of Theorem 1.2 as an elementary corollary.

Corollary 3.4. Suppose $f \in C(I, I)$ for which $\mathbf{h}(f)=0$. If $S(f)=P(f)$ and int $K=\emptyset$ for any simple system of $f$, then $\Omega:(C(I, I),\|\circ\|) \longrightarrow\left(K^{*}, H^{*}\right)$ is lower semicontinuous at $f$.

Proof. Let $\omega$ be an infinite $\omega$-limit set of $f$. Since $i n t K=\emptyset$ for any simple system of $f$, from Smital's Theorem one sees that there exists $\left\{\alpha_{n}\right\}_{n=1}^{\infty} \subseteq \Omega(f)$ so that $\alpha_{n} \subseteq S(f)$ for each $n$, and $\lim _{n \rightarrow \infty} \alpha_{n}=\omega$.

From examples found in $[\mathrm{B}],[\mathrm{TH}]$ as well as our earlier example, even a well behaved function $f \in C(I, I)$ need not be a point of lower semicontinuity of $\Omega:(C(I, I),\|\circ\|) \longrightarrow\left(K^{*}, H^{*}\right)$. We are able to show, however, that we can appropriately perturb any function $f \in C(I, I)$ to obtain a function $g$ in $C(I, I)$ with the property that a subset of $g$ 's stable periodic orbits do form an $\varepsilon$-net of $\Omega(f)$. Specifically, we prove the following.

Theorem 3.5. Let $f \in C(I, I)$. For any $\varepsilon>0$ there exists $g \in C(I, I)$ with $\left\{\omega_{1}, \omega_{2}, \ldots, \omega_{n}\right\}$ contained in $\Omega(g)$ so that $\omega_{i} \subseteq S(g)$ for any $1 \leq i \leq n$, $\|f-g\|<\varepsilon$ and $\left\{\omega_{1}, \omega_{2}, \ldots, \omega_{n}\right\}$ forms an $\varepsilon$-net of $\Omega(f)$.

It is worth noting that we may just as well take the sets $\omega_{i}$ contained in $\Omega(g)$ so that each $\omega_{i}$ is not only a stable periodic orbit of $g$, but that it also attracts trajectories generated by points found in a set of positive measure. This provides an interesting complement to the main results found in [JS], where the authors discuss the effects of random perturbations on the trajectories generated by a continuous function. Specifically, Jankova and Smital show that the $\omega$-limit sets generated by continuous maps of the interval subjected to random pointwise perturbations of their trajectories exhibit the following two paradoxical properties: (1) any trajectory, with probability 1, is transitive in a system of compact intervals forming a periodic orbit, but (2) except for those functions $f$ found in an exceptional nowhere dense set of $C(I, I)$, the length of 
these intervals is smaller than $\varepsilon$ whenever the perturbations are smaller than some $\delta(f)$.

We begin our development of Theorem 3.5 with the following lemma.

Lemma 3.6. Let $f \in C(I, I)$ with $\omega(x, f) \in \Omega(f)$. Then for any $\varepsilon>0$ there exists $N$ a nutural number so that $f^{n}(x) \in B_{\varepsilon}(\omega(x, f))$ whenever $n>N$.

Proof. Suppose, to the contrary, that no such $N$ exists. Then there exists a subsequence $\{n(k)\}_{k=1}^{\infty}$ in $\mathbb{N}$ so that $f^{n(k)}(x) \in[0,1] \backslash B_{\varepsilon}(\omega(x, f))$ for all $k$. Since $[0,1] \backslash B_{\varepsilon}(\omega(x, f))$ is compact, $\left\{f^{n(k)}(x)\right\}_{k=1}^{\infty}$ must contain at least one limit point in $[0,1] \backslash B_{\varepsilon}(\omega(x, f))$. But this implies that $\omega(x, f) \cap$ $[0,1] \backslash B_{\varepsilon}(\omega(x, f)) \neq \emptyset$, a contradiction.

As an immediate corollary we have the following.

Corollary 3.7. Let $f \in C(I, I)$ with $\omega \in \Omega(f)$. For any $\varepsilon>0$ there exists $x \in I$ so that $\omega(x, f)=\omega$ and $f^{n}(x) \in B_{\varepsilon}(\omega)$ for all $n=0,1,2,3, \ldots$.

The proof of Theorem 3.5 rests, in large part, on our following lemma, where we make good use of Corollary 3.7.

Lemma 3.8. Let $f \in C(I, I)$ with $\omega \in \Omega(f)$. For any $\varepsilon>0$ there exists $g \in C(I, I)$ and $\alpha \in \Omega(g)$ so that $\alpha \subseteq P(g),\|f-g\|<\varepsilon$ and $H(\omega, \alpha)<\varepsilon$.

Proof. If $\omega$ is itself periodic there is nothing to prove, so let us assume $\omega$ is infinite. Using Corollary 3.7, let $x \in I$ so that $\omega(x, f)=\omega$, and $\left\{f^{n}(x)\right\}_{n=0}^{\infty} \subseteq$ $B_{\frac{\varepsilon}{2}}(\omega)$. Now, take $\left\{x, y_{0}, y_{1}, \ldots, y_{n}\right\} \subseteq \omega$ to be an $\frac{\varepsilon}{2}$-net of $\omega(x, f)$, a compact subset of $[0,1]$. Since $\omega(x, f)=\omega$, there exists $N \in \mathbb{N}$ so that, for any $i \in\{0,1, \ldots, n\},\left|y_{i}-f^{k}(x)\right|<\frac{\varepsilon}{2}$ for some $1 \leq k \leq N$, and $\left|x-f^{N}(x)\right|<\varepsilon$. Choose a neighborhood $U$ of $f^{N-1}(x)$ so that $U \cap\left\{f^{n}(x)\right\}_{n=0}^{N}=f^{N-1}(x)$. Now, take $g \in C(I, I)$ so that

1. $f(x)=g(x)$ for $x \in I \backslash U$

2. $g\left(f^{N-1}(x)\right)=x$, and since $\left|x-f^{N}(x)\right|<\varepsilon$

3. $\|f-g\|<\varepsilon$.

It follows, then, that $\left\{x, f(x), f^{2}(x), \ldots, f^{N-1}(x)\right\}=\omega(x, g)$ is a periodic orbit of period $N$ for $g$. Since $\omega \subseteq B_{\frac{\varepsilon}{2}}\left(\left\{x, y_{0}, y_{1}, \ldots, y_{n}\right\}\right)$ and $\left\{x, y_{0}, y_{1}, \ldots, y_{n}\right\} \subseteq$ $B_{\frac{\varepsilon}{2}}(\omega(x, g))$, it follows that $\omega \subseteq B_{\varepsilon}(\omega(x, g))$. By our choice of $x$, we also have that $\left\{f^{n}(x)\right\}_{n=0}^{N-1} \subseteq\left\{f^{n}(x)\right\}_{n=0}^{\infty} \subseteq B_{\frac{\varepsilon}{2}}(\omega)$, so that $\omega(x, g) \subseteq B_{\frac{\varepsilon}{2}}(\omega)$. This implies that $H(\omega, \omega(x, g))<\varepsilon . \square$

If necessary, we may make arbitrarily slight perturbations in the function $g$ of Lemma 3.8 so as to take $\alpha$ to be a stable periodic orbit. This gives us the following stronger result as a corollary. 
Corollary 3.9. Let $f \in C(I, I)$ with $\omega \in \Omega(f)$. For any $\varepsilon>0$ there exists $g \in C(I, I)$ and $\alpha \in \Omega(g)$ so that $\alpha \subseteq S(g),\|f-g\|<\varepsilon$ and $H(\omega, \alpha)<\varepsilon$.

This puts us in a position to prove Theorem 3.5.

Proof of Theorem 3.5. Let $\left\{\alpha_{1}, \alpha_{2}, \ldots, \alpha_{n}\right\} \subseteq \Omega(f)$ be an $\frac{\varepsilon}{2}$-net of $\Omega(f)$. Using Corollary 3.9, to each $\alpha_{i}$ we can associate $g_{i} \in C(I, I)$ and $\omega_{i} \in \Omega\left(g_{i}\right)$ so that $\omega_{i} \subseteq S\left(g_{i}\right),\left\|f-g_{i}\right\|<\frac{\varepsilon}{2}$ and $H\left(\omega_{i}, \alpha_{i}\right)<\frac{\varepsilon}{2}$. Moreover, we may presume that $\omega_{i} \cap \omega_{j}=\emptyset$ whenever $i \neq j$ by taking a slight perturbation of our functions $g_{i}$ as necessary. Let $\delta>0$ so that $\delta<\min \left\{\frac{\varepsilon}{2}, \sigma\right\}$, where $\sigma=\min \left\{|x-y|: x \neq y\right.$ and $\left.x, y \in \cup_{i=1}^{n} \omega_{i}\right\}$. Now, take $g \in C(I, I)$ so that

1. $g(x)=g_{i}(x)$ for all $x \in \cup_{i=1}^{n} \omega_{i}$,

2. $g(x)=\max \left\{g_{i}(x): 1 \leq i \leq n\right\}$ for all $x \in I \backslash B_{\frac{\delta}{2}}\left(\cup_{i=1}^{n} \omega_{i}\right)$,

3. $\|f-g\|<\varepsilon$, and

4. $\omega_{i} \subseteq S(g)$ for $1 \leq i \leq n$.

If $\alpha \in \Omega(f)$, then $H\left(\alpha_{i}, \alpha\right)<\frac{\varepsilon}{2}$ for some $1 \leq i \leq n$. Since $H\left(\alpha_{i}, \omega_{i}\right)<\frac{\varepsilon}{2}$, it follows that $H\left(\omega_{i}, \alpha\right)<\varepsilon$ and that $\left\{\omega_{1}, \omega_{2}, \ldots, \omega_{n}\right\} \subseteq \Omega(g)$ is an $\varepsilon$-net of $\Omega(f)$.

\section{Upper Semicontinuity}

We say that our map $\Omega:(C(I, I),\|\circ\|) \longrightarrow\left(K^{*}, H^{*}\right)$ is upper semicontinuous at $f$ if $f_{n} \rightarrow f$ in $C(I, I)$ and $\Omega\left(f_{n}\right) \rightarrow L$ in $\left(K^{*}, H^{*}\right)$ implies $L \subseteq \Omega(f)$. It follows that the upper semicontinuity of $\Lambda:(C(I, I),\|\circ\|) \longrightarrow(K, H)$ is a necessary condition for the upper semicontinuity of $\Omega:(C(I, I),\|\circ\|) \longrightarrow\left(K^{*}, H^{*}\right)$. In [TH2] we characterize the points at which $\Lambda$ is upper semicontinuous as those functions $f$ for which $\Lambda(f)=C R(f)$. As an immediate corollary, then, we have the following.

Proposition 4.1. A necessary condition for the function $f$ to be a point of upper semicontinuity of the map $\Omega:(C(I, I),\|\circ\|) \longrightarrow\left(K^{*}, H^{*}\right)$ is that $\Lambda(f)=C R(f)$.

That the condition of Proposition 4.1 is not sufficient to insure that $f \in$ $C(I, I)$ is a point of upper semicontinuity of $\Omega:(C(I, I),\|\circ\|) \longrightarrow\left(K^{*}, H^{*}\right)$ follows from examples found in $[\mathrm{TH}]$. We now recall a pair of previously known results. The first is from $[\mathrm{BCl}]$, and the second can be found in $[\mathrm{TH}]$.

Proposition 4.2. Suppose $f \in C(I, I)$ with $\omega \in \Omega(f)$. If $F$ is any nonempty proper closed subset of $\omega$, then $F \cap \overline{f(\omega \backslash F)} \neq \emptyset$. 
Proposition 4.3. Suppose $f_{n} \rightarrow f, \omega_{n} \rightarrow L$ and $\omega_{n} \in \Omega\left(f_{n}\right)$ for each $n$. Then

1. $f(L)=L$

2. If $F$ is any nonempty proper closed subset of $L$, then $F \cap \overline{f(L \backslash F)} \neq \emptyset$.

Proposition 4.3 holds the key to our characterization of functions $f \in$ $C(I, I)$ at which $\Omega:(C(I, I),\|\circ\|) \longrightarrow\left(K^{*}, H^{*}\right)$ is upper semicontinuous. We know that any $\omega$-limit set of a continuous function $f$ satisfies both parts of the conclusion of Proposition 4.3 [BCl]; however, our function $f$ will be a point of upper semicontinuity of $\Omega:(C(I, I),\|\circ\|) \longrightarrow\left(K^{*}, H^{*}\right)$ only when these conditions characterize its $\omega$-limit sets. This is the content of Theorem 4.4 .

Theorem 4.4. The map $\Omega:(C(I, I),\|\circ\|) \longrightarrow\left(K^{*}, H^{*}\right)$ is upper semicontinuous at the function $f$ if and only if $L \in \Omega(f)$ whenever $L \in K$ for which $f(L)=L$ and $F \cap \overline{f(L \backslash F)} \neq \emptyset$ for any nonempty proper closed subset $F$ of $L$.

We make significant progress in proving Theorem 4.4 with the development of the following proposition.

Proposition 4.5. Let $f \in C(I, I)$ with $L \in K$ for which $f(L)=L$ and $F \cap \overline{f(L \backslash F)} \neq \emptyset$ for any nonempty proper closed subset $F$ of $L$. For any $\varepsilon>0$ there exists $g \in C(I, I)$ and $\omega \in \Omega(g)$ so that $\|f-g\|<\varepsilon$ and $H(\omega, L)<\varepsilon$.

Proof. Let $\varepsilon>0$. Since $f \in C(I, I), f$ is uniformly continuous on $I$, so that there exists $\delta_{1}>0$ with the property that $|f(x)-f(y)|<\varepsilon$ whenever $|x-y|<$ $\delta_{1}$. Choose $\delta$ so that $0<\delta<\min \left\{\delta_{1}, \varepsilon\right\}$, and take $\left\{x_{1}, x_{2}, \ldots, x_{n}\right\} \subseteq L$ to be a $\delta$-net for $L$. It suffices to perturb $f$ to get a function $g \in C(I, I)$ possessing a periodic attractor $\omega$ so that $\|f-g\|<\varepsilon$ and $\left\{x_{1}, x_{2}, \ldots, x_{n}\right\} \subseteq \omega \subseteq \cup_{i=1}^{n} B_{\delta}\left(x_{i}\right)$, as this implies $H(\omega, L)<\varepsilon$. That this is possible follows from our hypothesis that $F \cap \overline{f(L \backslash F)} \neq \emptyset$ for any nonempty proper closed subset $F$ contained in $L$. In particular,

1. let $F=L \backslash B_{\delta}\left(x_{i}\right)$ to see that there exists $x \in B_{\delta}\left(x_{i}\right)$ such that $f(x) \in$ $B_{\delta}\left(x_{j}\right)$ for some $j \neq i$, for any $i=1,2, \ldots, n$;

2. let $F=L \backslash \cup_{j \neq i} B_{\delta}\left(x_{j}\right)$ to see that there exists $x \in B_{\delta}\left(x_{j}\right)$ for some $j \neq i$ so that $f(x) \in B_{\delta}\left(x_{i}\right)$, for any $i=1,2, \ldots, n$, and

3. let $S \subseteq\{1,2, \ldots, n\}$ with $F=L \backslash \cup_{i \in S} B_{\delta}\left(x_{i}\right)$ to see that there exists $x \in \cup_{i \in S} B_{\delta}\left(x_{i}\right)$ so that $f(x) \in B_{\delta}\left(x_{j}\right)$ for some $j \in\{1,2, \ldots, n\} \backslash S$. 
With Proposition 4.5, a proof of Theorem 4.4 follows easily. Proof of Theorem 4.4. Suppose $\Omega:(C(I, I),\|\circ\|) \longrightarrow\left(K^{*}, H^{*}\right)$ is upper semicontinuous at $f$, and $L \in K$ for which $f(L)=L$ and $F \cap \overline{f(L \backslash F)} \neq \emptyset$ for any nonempty proper closed subset $F$ of $L$. By Proposition 4.5, there exists $\left\{f_{n}\right\} \subseteq C(I, I)$ with $\omega_{n} \in \Omega\left(f_{n}\right)$ for any $n$ so that $\lim _{n \rightarrow \infty} f_{n}=f$ and $\lim _{n \rightarrow \infty} \omega_{n}=L$. Since $\Omega$ is upper semicontinuous at $f$, it follows that $L \in \Omega(f)$.

Now, suppose that $L \in \Omega(f)$ whenever $L \in K$ for which $f(L)=L$ and $F \cap \overline{f(L \backslash F)} \neq \emptyset$ for any nonempty proper closed subset $F$ of $L$. Let $\left\{f_{n}\right\} \subseteq C(I, I)$ with $\omega_{n} \in \Omega\left(f_{n}\right)$ for any $n$ so that $\lim _{n \rightarrow \infty} f_{n}=f$ and $\lim _{n \rightarrow \infty} \omega_{n}=L^{*}$. Since $f\left(L^{*}\right)=L^{*}$ and $F \cap \overline{f\left(L^{*} \backslash F\right)} \neq \emptyset$ for any nonempty proper closed subset $F$ of $L^{*}$ by Proposition 4.3 , it follows that $L^{*} \in \Omega(f)$, so that $\Omega:(C(I, I),\|\circ\|) \longrightarrow\left(K^{*}, H^{*}\right)$ is upper semicontinuous at $f$.

We turn our attention to the development of a few corollaries of Theorem 4.4. These shed considerable light on the structure of those continuous functions that are points of upper semicontinuity of $\Omega:(C(I, I),\|\circ\|) \longrightarrow$ $\left(K^{*}, H^{*}\right)$. In particular, our first two corollaries show that, in many instances, wandering intervals preclude upper semicontinuity.

Corollary 4.6. Let $f \in C(I, I)$ for which $\mathbf{h}(f)=0$. If int $K \neq \emptyset$ for some simple system of $f$, then $\Omega:(C(I, I),\|\circ\|) \longrightarrow\left(K^{*}, H^{*}\right)$ is not upper semicontinuous at $f$.

Proof. Since $f(K)=K$ and $F \cap \overline{f(K \backslash F)} \neq \emptyset$ for any nonempty proper closed subset $F$ of $K$, it suffices to show that $K \notin \Omega(f)$. But this follows from the fact that every $\omega$-limit set of a function with zero topological entropy must be nowhere dense.

Corollary 4.7. Let $f \in C(I, I)$ with positive topological entropy, and take $X \subset I$ so that $f^{n}(X)=X$, and $f^{n} \mid X$ is semiconjugate to the shift operator $\sigma$ on two symbols, for some natural number $n$. If $X$ possesses a component with nonempty interior, then $\Omega:(C(I, I),\|\circ\|) \longrightarrow\left(K^{*}, H^{*}\right)$ is not upper semicontinuous at $f$.

Proof. We begin by noting that it suffices to show that $\Omega:(C(I, I),\|\circ\|) \longrightarrow$ $\left(K^{*}, H^{*}\right)$ is not upper semicontinuous at $f^{n}$. Since $f^{n}(X)=X$ and $F \cap$ $\overline{f^{n}(X \backslash F)} \neq \emptyset$ for any nonempty proper closed subset $F$ of $X$, we want to show that $X \notin \Omega\left(f^{n}\right)$ for our conclusion to follow. Since $X$ possesses a component with nonempty interior, $X$ must possess infinitely many components with nonempty interior. That $X$ cannot be an $\omega$-limit set now follows from the characterization of $\omega$-limit sets for continuous functions found in Theorem 2.4 . 
Our next corollary reflects a certain stability in the algebraic structure of $\Omega(f)$ whenever $\Omega:(C(I, I),\|\circ\|) \longrightarrow\left(K^{*}, H^{*}\right)$ is upper semicontinuous at $f$.

Corollary 4.8. Let $f \in C(I, I)$. If $\Omega:(C(I, I),\|\circ\|) \longrightarrow\left(K^{*}, H^{*}\right)$ is upper semicontinuous at $f$, then $\omega_{1} \cup \omega_{2} \in \Omega(f)$ whenever $\omega_{1}$ and $\omega_{2}$ are elements of $\Omega(f)$, and $\omega_{1} \cap \omega_{2} \neq \emptyset$.

Proof. Since $f\left(\omega_{i}\right)=\omega_{i}$ and $F \cap \overline{f\left(\omega_{i} \backslash F\right)} \neq \emptyset$ for any nonempty proper closed subset $F$ of $\omega_{i}$, for both $i=1,2$, the same is true their union. Our conclusion now follows from Theorem 4.4.

We conclude this section with an application of Corollary 4.8 that solves an open problem from [TH2].

Example 4.9. Define the double hat map $h:[-1,1] \longrightarrow[-1,1]$ so that $h(-1)=h(0)=h(1)=0, h\left(\frac{1}{2}\right)=-h\left(-\frac{1}{2}\right)=1$, and $h$ is extended linearly to all of $[-1,1]$. Then $\Omega:(C([-1,1],[-1,1]),\|\circ\|) \longrightarrow\left(K^{*}, H^{*}\right)$ is not upper semicontinuous at $h$ even though $\Lambda:(C([-1,1],[-1,1]),\|\circ\|) \longrightarrow(K, H)$ is continuous there, and $P(h)=S(h)$.

Proof. We begin by noting that both $\omega_{1}=\{0\} \cup \cup_{n=0}^{\infty}\left\{\frac{1}{2^{n}}\right\}$ and $\omega_{2}=$ $\{0\} \cup \cup_{n=0}^{\infty}\left\{-\frac{1}{2^{n}}\right\}$ are homoclinic $\omega$-limit sets of $h:[-1,1] \longrightarrow[-1,1]$. Since $h([0,1])=[0,1]$ and $h([-1,0])=[-1,0]$, it follows that $\omega_{1} \cup \omega_{2}$ is not an $\omega$-limit set of $h$, so that $\Omega:(C([-1,1],[-1,1]),\|\circ\|) \longrightarrow\left(K^{*}, H^{*}\right)$ cannot be upper semicontinuous there.

\section{Conclusions}

While we have made some progress in understanding the overall behavior of $\Omega:(C(I, I),\|\circ\|) \rightarrow\left(K^{*}, H^{*}\right)$ with our consideration of semicontinuity, obvious questions remain. In particular, how can we improve Proposition 3.3 so as to develop a characterization of those functions $f \in C(I, I)$ at which $\Omega:(C(I, I),\|\circ\|) \rightarrow\left(K^{*}, H^{*}\right)$ is lower semicontinuous? A major step in developing such a characterization would be an answer to the following query: Let $\varepsilon>0$. What conditions on $f \in C(I, I)$ and $\omega \in \Omega(f)$ insure the existence of a periodic $\omega$-limit set $\alpha$ in $\Omega(f)$ so that $H(\omega, \alpha)<\varepsilon$ ? An answer to this query would not only help us characterize those continuous functions at which $\Omega:(C(I, I),\|\circ\|) \rightarrow\left(K^{*}, H^{*}\right)$ is lower semicontinuous, but may also provide significant insight into $\Omega$ 's continuity structure vis-a-vis the chaotic nature of $f$ in $C(I, I)$. 


\section{References}

[1] S. J. Agronsky, A. M. Bruckner, J. G. Ceder and T. L. Pearson, Structure of w-limit sets for continuous functions, Real Anal. Ex. 15(1989), 483510 .

[2] L. Block and W. Coppel, Dynamics in one dimension, Lecture Notes in Mathematics, vol. 1513, Springer-Verlag, 1991.

[3] A. Blokh, A. M. Bruckner, P. D. Humke and J. Smital, The space of $\omega$-limit sets of a continuous map of the interval, Trans. Amer. Math. Soc. 348 (1996), 1357-1372.

[4] A.M. Bruckner, Stability in the family of $\omega$-limit sets of continuous self maps of the interval, Real Anal. Ex. 22 (1997), 52-57.

[5] A. M. Bruckner, J. B. Bruckner and B. S. Thomson, Real analysis, Prentice-Hall, 1997.

[6] A. M. Bruckner and J. G. Ceder, Chaos in terms of the map $x \longmapsto \omega(x, f)$, Pac. J. Math. 156 (1992), 63-96.

[7] R. L. Devaney, Chaotic dynamical systems, Benjamin/Cummings Publ. Co., 1986.

[8] V. Fedorenko, A. Sarkovskii and J. Smital, Characterizations of weakly chaotic maps of the interval, Proc. Amer. Math. Soc. 110 (1990), 141-148.

[9] K. Jankova and J. Smital, Maps with random perturbations are generically not chaotic, Intl. J. Bifur. and Chaos 5 (1995), 1375-1378.

[10] D. Preiss and J. Smital, A characterization of nonchaotic maps of the interval stable under small perturbations, Trans. Amer. Math. Soc. 313 (1989), 687-696.

[11] J. Smital, Chaotic functions with zero topological entropy, Trans. Amer. Math. Soc. 297 (1986), 269-282.

[12] T. H. Steele, Iterative stability in the class of continuous functions, Real Anal. Ex. 24 (1999), 765-780.

[13] T. H. Steele, Continuity structure of $f \rightarrow \cup_{x \in I} \omega(x, f)$ and $f \rightarrow$ $\{\omega(x, f): x \in I\}$, Real Anal. Ex. 25 (1999), 421-428. 